\title{
Performance Analysis of Chain Code DESCRIPTOR FOR HAND SHAPE ClASSIFICATION
}

\author{
Kshama Fating $^{1}$ and Archana Ghotkar ${ }^{2}$ \\ ${ }^{1,2}$ Department of Computer Engineering, Pune Institute of Computer Technology, \\ Pune, India
}

\begin{abstract}
Feature Extraction is an important task for any Image processing application. The visual properties of any image are its shape, texture and colour. Out of these shape description plays important role in any image classification. The shape description method classified into two types, contour base and region based. The contour base method concentrated on the shape boundary line and the region based method considers whole area. In this paper, contour based, the chain code description method was experimented for different hand shape.
\end{abstract}

The chain code descriptor of various hand shapes was calculated and tested with different classifier such as k-nearest- neighbour (k-NN), Support vector machine (SVM) and Naive Bayes. Principal component analysis (PCA) was applied after the chain code description. The performance of SVM was found better than $k-N N$ and Naive Bayes with recognition rate $93 \%$.

\section{KEYWORDS}

Feature extraction, Chain code, $k-N N$, SVM, Naive Bayes

\section{INTRODUCTION}

With the development of information technology, the use of human-computer interaction (HCI) based application increased. There are many applications of HCI such as control mechanical system, computer game, interacting with visualization system. These applications use interface based on visual input. Advantage of visual input is, it is possible to communicate any device without any physical contact. There are many visual inputs are use for HCI application such as facial expression, speech command, hand shape. We have used hand shape for classification which can be used in any HCI application. With different modalities such as lips, eye movement, facial expression, speech command, hand shape is good in noisy environment.

An image important features are shape, colour and texture. Shape is an important feature of an object. It contains more facts about an object than other features, such as colour. The shape can be described into two different methods. The first method uses boundary features and the other method uses region features to describe the shape. Boundary features are extracted from the boundary of the shape like perimeter and corners, while regional features are extracted from the region occupied by the shape such as the area [1]. Boundary features can be found by applying contour detection techniques. Contour detection has played important role in recognizing the shape of any object. In order to classify any shape, the shape has to describe by certain method. We have used, contour based, chain code description method for hand shape classification. This paper focuses on representation of a shape using chain code and classified shape based on their chain code. 


\section{RELATED WORK}

The chain code descriptor used by many authors for various applications. The survey of shape representation techniques, chain code with the application and dataset are presented in Table 1.

Table 1.Survey on Chain code techniques for representation and recognition of shape

\begin{tabular}{|c|c|c|c|}
\hline Application & Dataset & Descriptor use & Reference Number \\
\hline $\begin{array}{l}\text { To detect object in an } \\
\text { image and video }\end{array}$ & $\begin{array}{l}\text { Static images and } \\
\text { Real time video }\end{array}$ & $\begin{array}{l}\text { Freeman chain } \\
\text { code }\end{array}$ & [2] \\
\hline $\begin{array}{l}\text { Recognition of irregular } \\
\text { object }\end{array}$ & $\begin{array}{l}\text { Alphabets from A } \\
\text { to } Z \text { and irregular } \\
\text { shapes }\end{array}$ & $\begin{array}{l}\text { Chain code } \\
\text { histogram }\end{array}$ & {$[3]$} \\
\hline $\begin{array}{l}\text { Represent and } \\
\text { recognition of shape for } \\
\text { children education. }\end{array}$ & $\begin{array}{l}\text { Basic Shapes- } \\
\text { Rectangle, Square, } \\
\text { Circle etc. }\end{array}$ & $\begin{array}{l}\text { Freeman chain } \\
\text { code }\end{array}$ & [4] \\
\hline To recognize digit & Digits from 0-9 & $\begin{array}{l}\text { Freeman chain } \\
\text { code }\end{array}$ & [5] \\
\hline $\begin{array}{l}\text { To represent shapes } \\
\text { composed of triangular, } \\
\text { rectangular, and } \\
\text { hexagonal cells }\end{array}$ & $\begin{array}{l}\text { Thinned binary } \\
\text { images, namely } \\
\text { cube, stair, and } \\
\text { rectangle. }\end{array}$ & Vertex Chain Code. & [6] \\
\hline Recognition of Objects. & $\begin{array}{l}\text { Binary images of } \\
\text { tree leaves and } \\
\text { airplanes images }\end{array}$ & Vertex Chain Code. & [7] \\
\hline
\end{tabular}

\section{DATA SET}

We have used total 300 images, 200 images for training and 100 images for classification. Figure 1 shows the dataset used for training and classification. The training dataset contains hand shapes with classification labels.

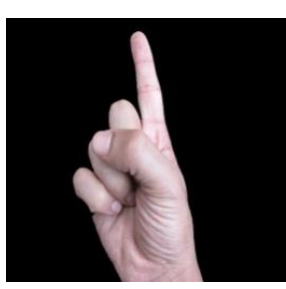

Class 1

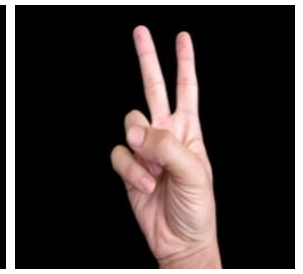

Class 2

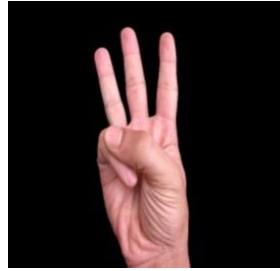

Class 3

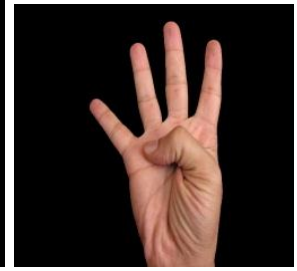

Class 4

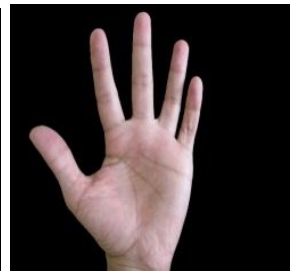

Class 5

Figure 1. Sample Shapes 


\section{Methodology}

The goals of this paper are threefold: first, it described the shape using chain code descriptor; second, reduce dimensions of training and testing data with principal component analysis and third, classifies shape in giving classes using the classifiers. Five classes of hand shape were considered with a raised finger. Figure 2 shows the proposed model for shape representation and classification.

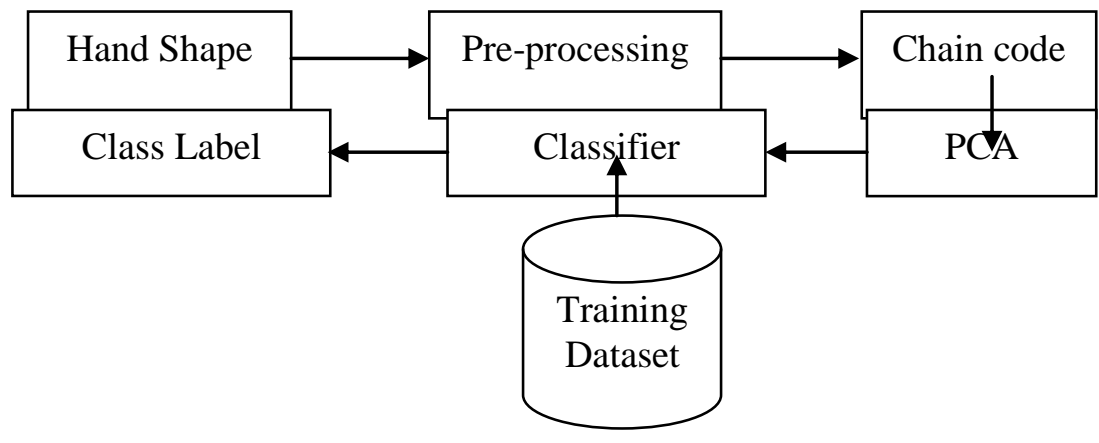

\subsection{Pre-processing}

Figure 2. Classification Process

In pre-processing, image is segmented with threshold 80. After segmentation, contour was found. We have used the polygon approximation, to approximate polygonal curves with the specified precision. Figure 3 shows, pre-processing of input image for classification.

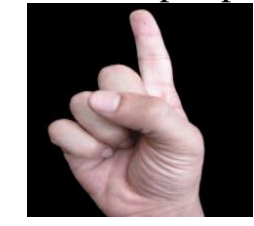

(a)

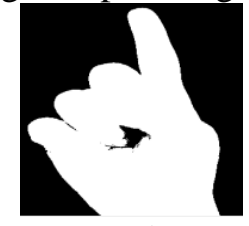

(b)

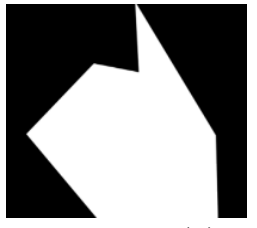

(c)

Figure 3. (a)Original Image (b) segmented image (c) polygon approximation

\subsection{Chain Code}

The shape can be well represented by boundary. Chain code provides a storage efficient representation for boundary of an object [8]. There are three techniques of chain code descriptor to represent the shape.

\subsubsection{Freeman Chain Code}

Chain code represents an object boundary by a connected sequence of straight line segments of specified length and direction. This straight line segment is a sequence of integer.

Let sequence of integer $X=\left\{x_{0}, x 2 \ldots n_{-1}\right\}$

$\mathrm{x}_{0}$ and $\mathrm{x}_{\mathrm{n}-1}$ are called start and terminate point

Having Each $x_{i}$ from the set $\{0,1 \ldots \ldots$. 1$)$

For 8 directional chain code $n=8$

For 4 directional chain code $n=4$

For $\mathrm{i}=1 \ldots \mathrm{N}$

$\mathrm{N}$ is total number of integer 

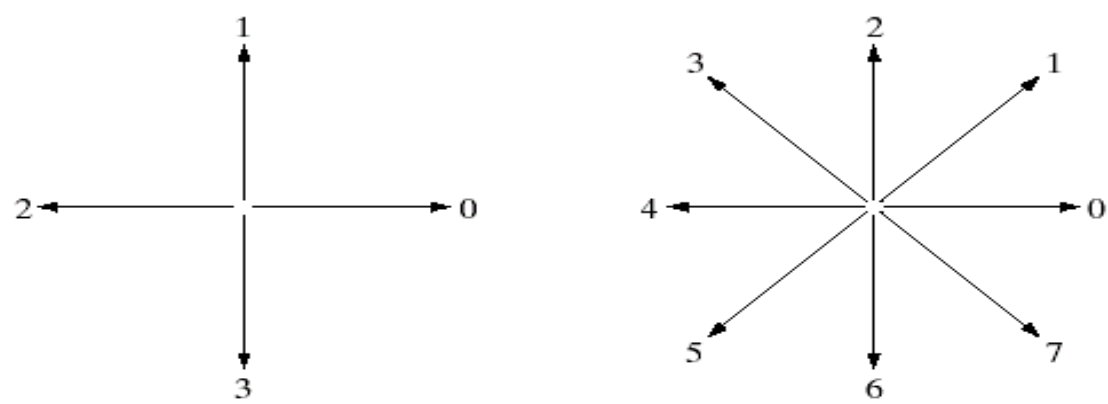

Figure 4.4 and 8 directional chain code

Figure 4 shows, 4 and 8 directional chain code to find the direction code. To find the chain code, first object need to be scanned from left to right. After finding the starting point of an object boundary is traverse till end pixel. Direction code is identified and store into array or list.

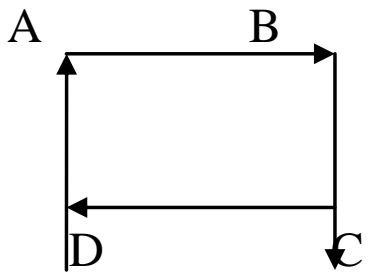

Figure 5. Original object

For the original object shown in Figure 5, the starting point is at the A. The 4 directional chain code of the object is $\mathbf{0 3 2 1}$ and 8 directional chain code of the object is $\mathbf{0 6 4 2}$. Starting point of an object defines the chain code. If we change the scale or rotation affects the chain code. Hence the chain code should be normalized. Basic chain code is only translation invariant, for rotationally invariant differential chain code is used. Scale invariance obtains by changing the size of sampling grid.

\section{Differential chain code:}

Differential chain code is obtained from first difference of chain code. The first difference of chain code is obtained by taking two numbers of chain code and calculating the number of transitions required to reach second number from the first number in counter clockwise direction [1]. The first difference is rotational invariant. The shape number is obtained after normalization of differential chain code. For minimum magnitude of shape number normalization use.

Normalization: For normalization treats a chain code as a circular sequence and redefine the starting point so that the resulting sequence of numbers contain a minimum integer.

Steps for Normalization:

1. Find chain code of object

2. Redefine the starting point-take last number at first position

3. Find first difference of chain code

4. Find the shape Number

A shape shown in Figure 5, using eight direction chain code. 
Chain code of shape: $\quad 0642$

Redefine starting point: 20642

Find first difference : 2-0, 0-6, 6-4, 4-2

Shape Number : $\quad \mathbf{6 6 6 6 6}$

D

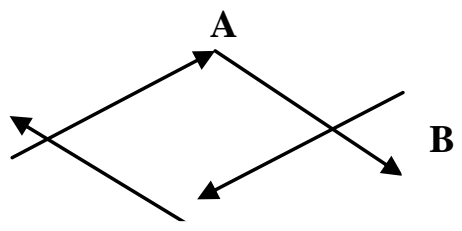

C

Figure 6. Rotation of original object

Figure 6 is shown; the original object is rotated in the right direction. Differential chain code is rotational invariant so; shape number of rotated object is same as the original object.

Chain code of rotated object: $\mathbf{7 5 3 1}$

Redefine starting point:

17531

Find first difference : 1-7,7-5,5-3,3-1

6666

Shape Number : $\quad \mathbf{6 6 6 6 6}$

Shape number of the original object is same as rotated object. So to get rotational invariant differential chain code was used.

\section{Resampling chain code:}

Chain code is sensitive to noise. This problem is solved by resembling the boundary by selecting larger grid spacing. Scale invariance obtains by changing the size of sampling grid.

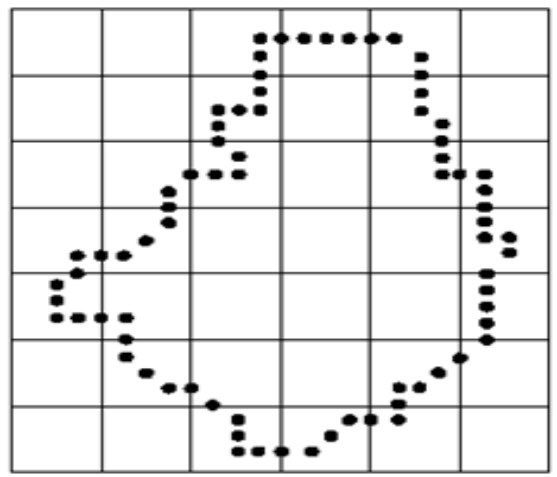

Figure 7. Original Image Points

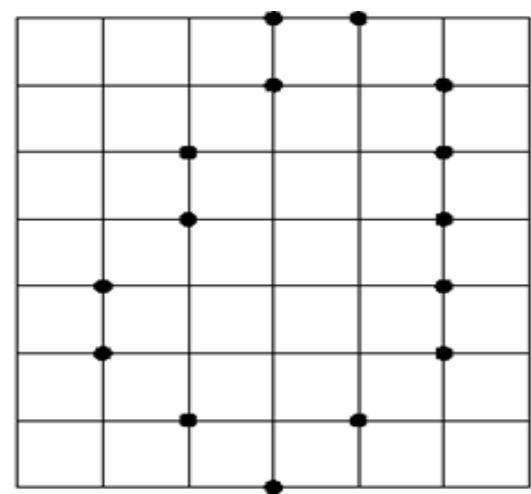

Figure 8. Result of Resampling

Figure 7 [9] shows original image points. Figure 8 shows resampling of original image points which contains less noise than the original image. Using resampling we can achieve scale invariant in case of freeman chain code.

In Freeman chain code method the object can be reconstructed from its chain code representation. 


\subsubsection{Vertex Chain code}

Vertex chain code improves the chain code efficiency. The Vertex chain code is based on the numbers of cell vertices which are in touch with the bounding contour of the shape [10]. In the vertex chain code only three elements use to represent the shape 1,2 and 3. Figure 9 shows, vertex chain code to identify the elements for shape representation.

If two vertices touch then

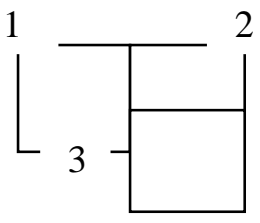

Figure 9. Vertex Chain Code

Element $=1$

If three vertices touch then

Element $=2$

If four vertices touch then

Element $=3$

\subsubsection{Chain code Histogram}

The chain code histogram $(\mathrm{CCH})$ is meant to group together objects that look similar to a human observer. $\mathrm{CCH}$ has made by counting the number of each kind of steps in Freeman chain code representation of the contour.

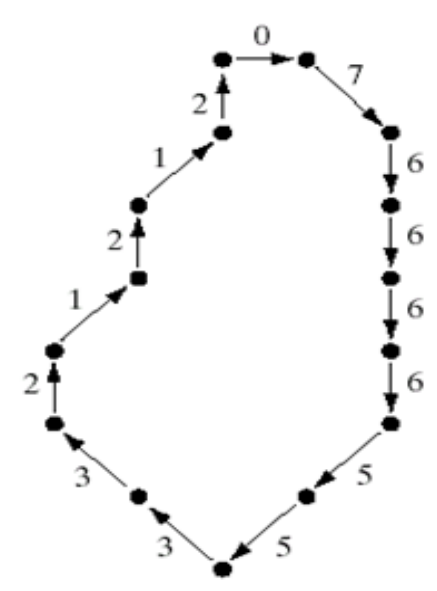

(a)

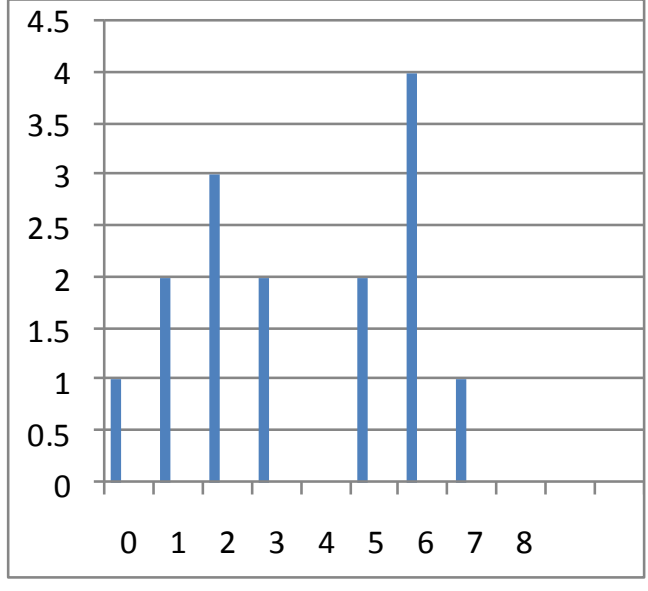

(b)

Figure 10. (a) Freeman Chain code: 076666553321212 (b) Chain code Histogram Figure 10 [9] shows freeman chain code and its histogram.

In this paper freeman chain code descriptor was used because it is translation, rotation, scale invariant and its simplicity.

\subsection{Feature Reduction and Classification}

Dimension reduction is the second step of the proposed model. We have used PCA for dimension reduction, which gives features vector for classification. 
A third step of proposed model is classification where test data compared with training dataset. There are several methods have been proposed for object recognition and classification. Manuele Bicego and Vittorio Murino used Hidden Markova model for classification [11]. This is sensitive to noise and cannot recognize the object with holes. Serge Belongie, Jetendra Malik and Jan Puzicha used shape contexts for object recognition [12]. Haibin Ling and David Jacobs designed classification method based on inner distance [13]. Thiago R. Trigo and Sergio Roberto M. Pellegrino proposed classification system using the geometric shape descriptors for hand gesture classification [14]. We have used k-NN and SVM and Naive Bayes classifiers for classification.

\subsection{1. k-Nearest Neighbour}

This is simple discriminative classifier. Training data are stored with labels. Thereafter a test data was classified according to the majority vote of its k-Nearest other data points. Here nearness is check according to Euclidean distance and the value of $\mathrm{k}$ is 5. Classification results using k-NN are given in Figure 11.

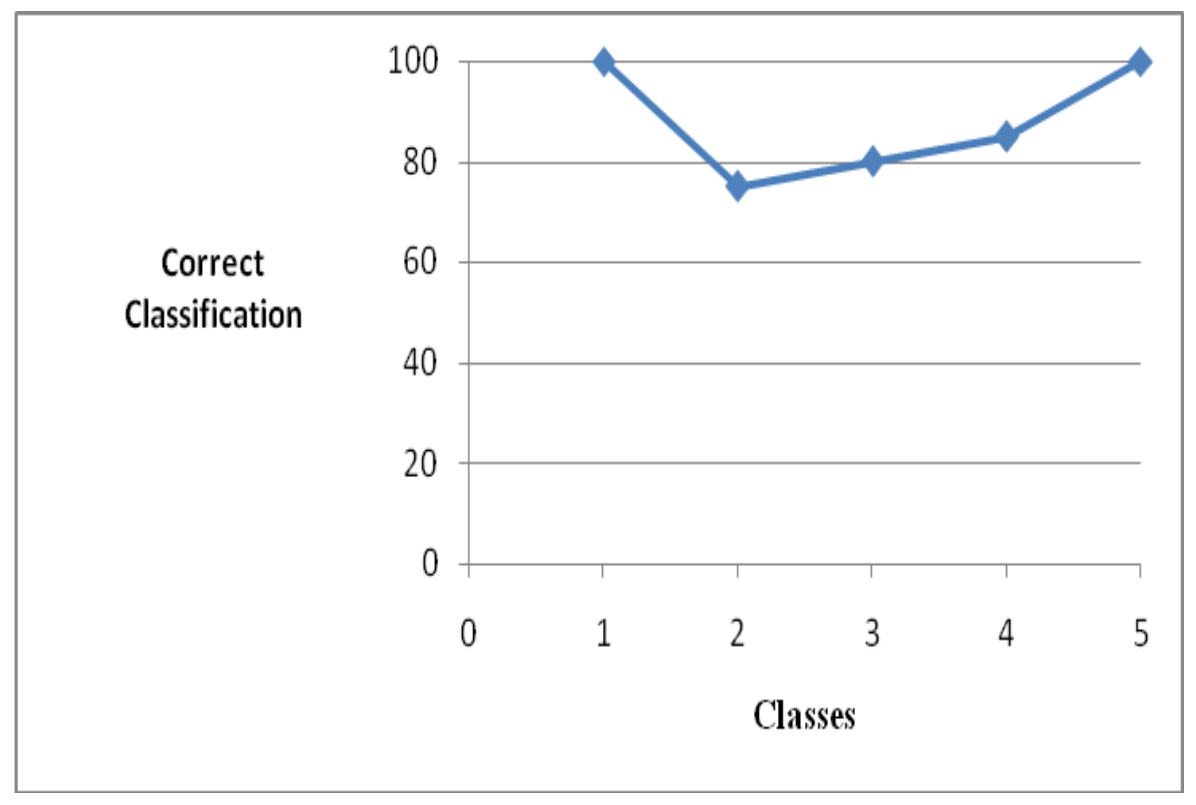

Figure 11. Classification Results of k-NN classifier

\subsubsection{Support Vector Machine}

Support vector machine is a discriminative classifier formally defined by the separating hyperplan. SVM uses the trained data to define the hyperplane which classify the input test data [15]. SVM classifies linearly separable data which contain two classes as well as non linear data which contains multiple classes. Classification results using SVM are given in Figure 12. 
International Journal of Computer Graphics \& Animation (IJCGA) Vol.4, No.2, April 2014

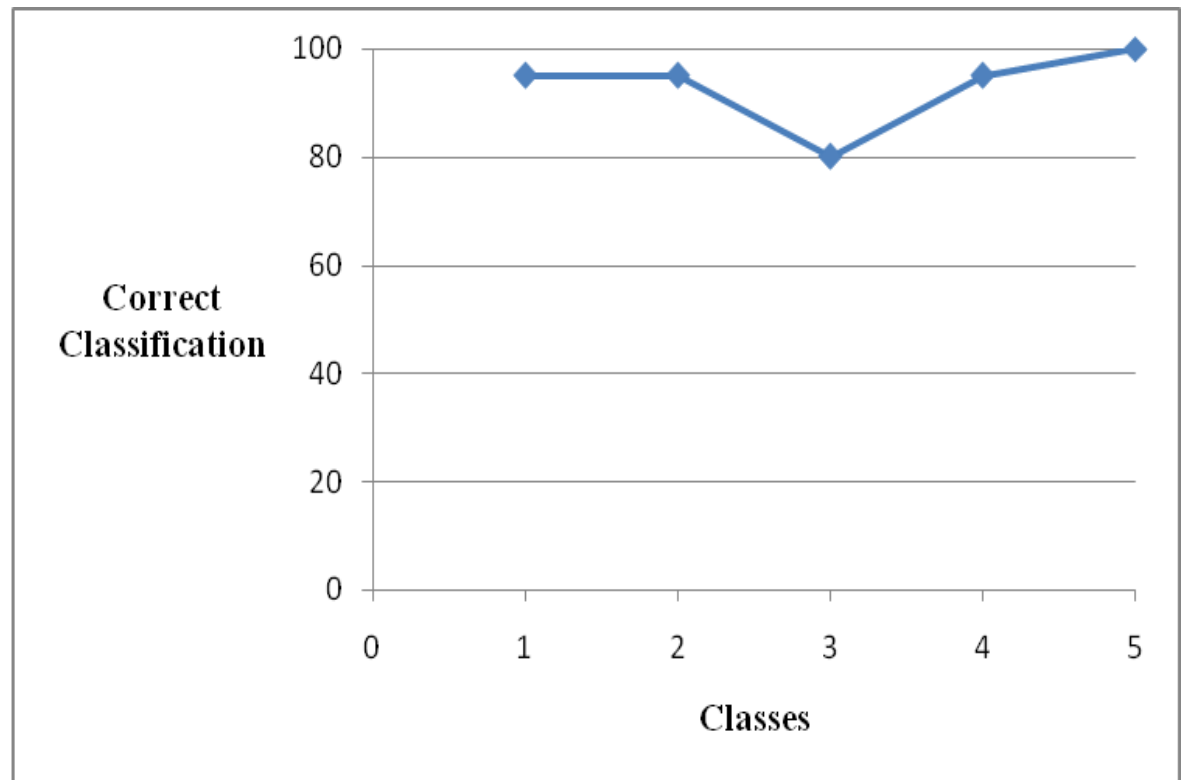

\subsubsection{Naive Bayes}

Figure 12. Classification Results of SVM classifier

The Bayesian classification represents a supervised learning method as well as statistical classification method. It is based on Bayesian theorem. It is particularly suited for large dimensional data [15].

Let given features are $X_{1}, X_{2} \ldots X n$, Predict the label $Y$

$$
\begin{aligned}
& X_{1, \ldots ., X n} \in\{0,1\} \text { (Black vs. White pixels) } \\
& Y \in\{1,2,3,4,5\}
\end{aligned}
$$

Use Bayes rule:
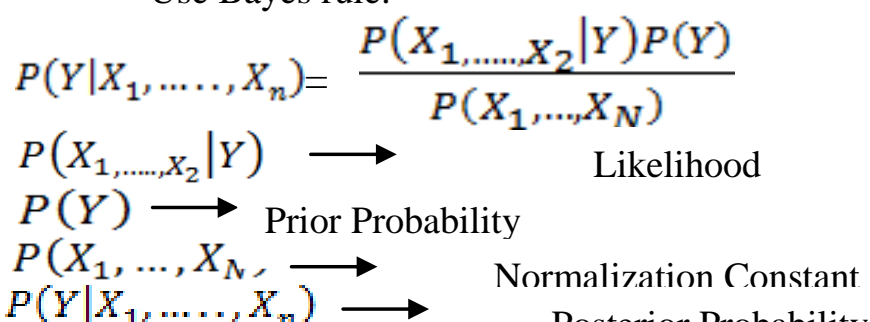

Posterior Probability

During testing, posterior probability of all classes is calculated, and prediction of class based on which one is greater. Classification results using Naive Bayes are given in Figure 13. 
International Journal of Computer Graphics \& Animation (IJCGA) Vol.4, No.2, April 2014

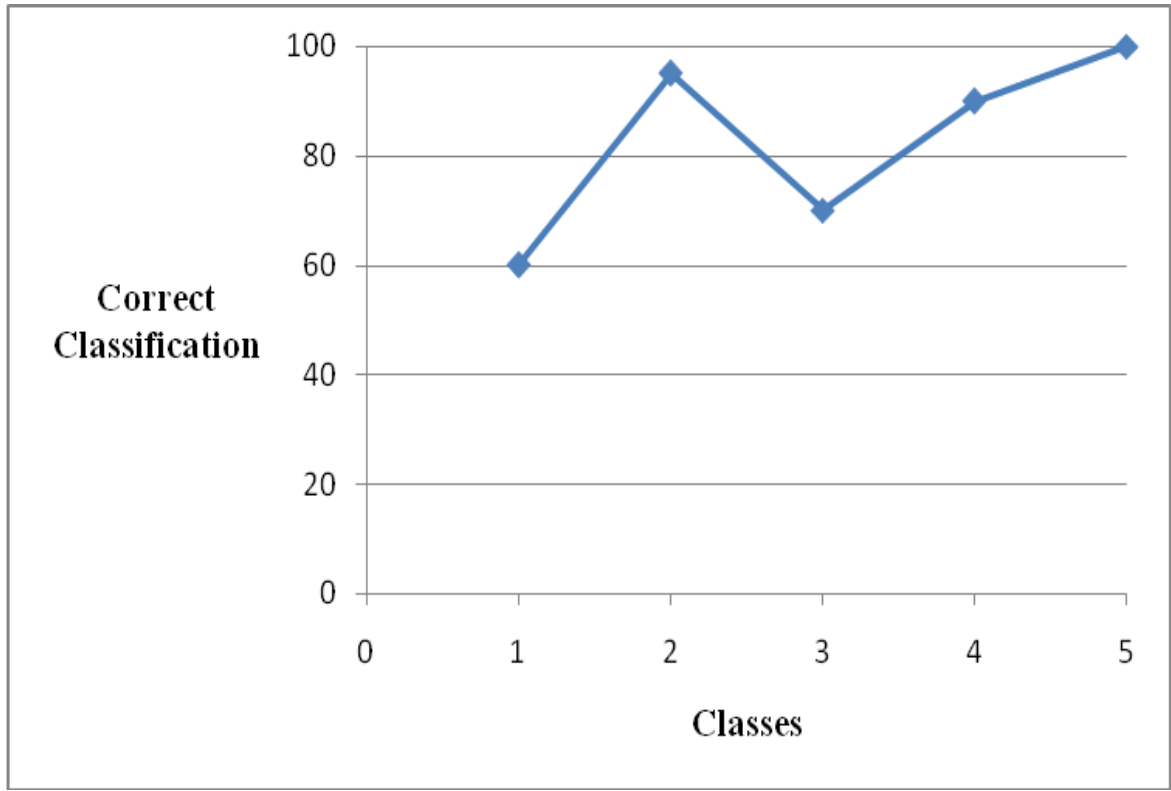

Figure 13. Classification Results of Naïve Bayes classifier

\section{EXPERIMENT RESULTS}

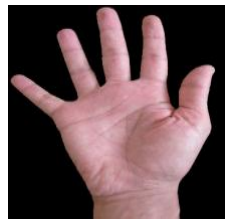

Input Shape

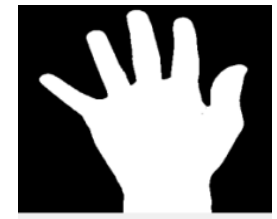

Preprocessing

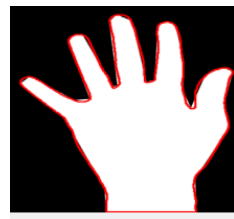

Chain code

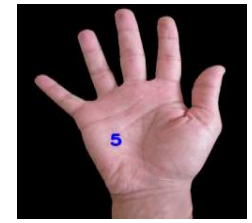

Classification

Figure 14. Classification of Input shape

Figure 14 shows classification process of input hand shape. In pre-processing colour image converted into binary and shape is represented by chain code. In Classification, chain code was tested with three classifiers which result into class label.

Table 2. Classification Results

\begin{tabular}{|c|c|c|c|c|}
\hline Hand Images & $\begin{array}{c}\text { Total Test } \\
\text { Images }\end{array}$ & $\begin{array}{c}\text { k-NN } \\
\text { \% }\end{array}$ & $\begin{array}{c}\text { SVM } \\
\%\end{array}$ & $\begin{array}{c}\text { Naive Bayes } \\
\%\end{array}$ \\
\hline (I) & 20 & 100 & 95 & 60 \\
\hline II & 20 & 75 & 95 & 95 \\
\hline
\end{tabular}


International Journal of Computer Graphics \& Animation (IJCGA) Vol.4, No.2, April 2014

\begin{tabular}{|c|c|c|c|c|}
\hline & 20 & 85 & 95 & 90 \\
\hline & 20 & 100 & 100 & 100 \\
\hline Average & 100 & 88 & 93 & 83 \\
\hline
\end{tabular}

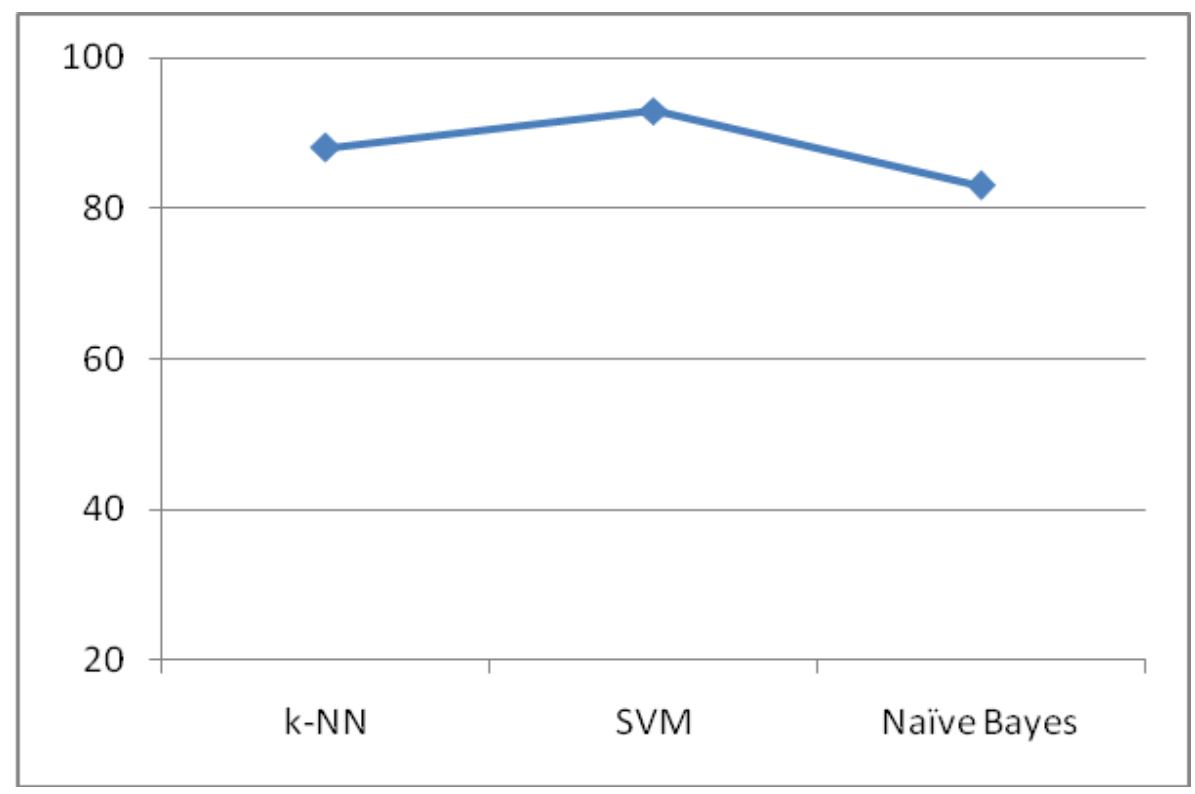

Figure 15. Classification Results

\section{ConClusion}

Here performance analysis of chain code descriptor with various classifiers is presented. We have tested 100 hand shapes with k-NN, SVM and Naive Bayes classifiers. Experimental results are given in the Table 2 shows performance of classifier with chain code. Figure 15 shows details of classification results. Classification of chain code with k- nearest-neighbour was $88 \%$, with support vector machine was $93 \%$ and with Naive Bays was $83 \%$. Experimental results show that chain code gives better performance with SVM classifier. For more training dataset performance of classifiers can surely increase. Here classes are chosen which will be useful for any HCI application.

\section{REFERENCES}

[1] S.Sridhar,(2012) "Digital Image Processing", Oxford University Press.

[2] R.S.Vaddi1, L. N.P.Boggavarapu1, H. D.Vankayalapati, K. R. Anne,(2011) "Contour detection using freeman chain code and approximation methods for real time object detection", AJCSIT.

[3] Jukka Iivarinen, Ari Visa,(1996) “Shape Recognition of Irregular Objects”, Proc. SPIE 2904.

[4] Dr. Azzam Talal Sleit, Rahmeh Omar Jabay, (2006) "A Chain Code Approach for Recognizing Basic Shapes", CSIT.

[5] Pulipati Annapurna, Sriraman Kothuri, Srikanth Lukka, (2013) "Digit Recognition Using Freeman Chain Code",JAIEM. 
International Journal of Computer Graphics \& Animation (IJCGA) Vol.4, No.2, April 2014

[6] Lili Ayu Wulandhari,Habibolah Haron,Ariffin Mohammad,(2008)“The Mapping Algorithm of Rrectangular Vertex Chain Code from Thinned Binary Image", International Conference on Engineering Optimization.

[7] Abdel-Badeeh M. Salem, Adel A. Sewisy, Usama A. Elyan,(2005) "A Vertex Chain Code Approach for Image Recognition", ICGST-GVIP Journal, Volume 5, Issue3.

[8] Yang Mingqiang, Kpalma Kidiyo, Ronsin Joseph,(2008) " A survey of shape feature extraction techniques", Pattern Recognition, Peng-Yeng Yin (Ed.)43-90.

[9] Rafael C. Gonzalez,Richard E.Woods,(1998) "Digital Image Processing”, Addison Wesley.

[10] Bribiesca E,(1999) “A New Chain Code. Pattern Recognition”, Vol. 32, issue 2, 235-25.

[11] Manuele Bicego, Vittorio Murino,(2004) "Investigating Hidden Markov Models' Capabilities in 2D Shape Classification”,IEEE Transaction on Pattern Analysis and Machine Intelligence,Vol.26, NO.2.

[12] Serge Belongie, Jetendra Malik and Jan Puzicha,(2002) "Shape matching and Object recognition using Shape Context", IEEE Transaction on Pattern Analysis and Machine Intelligence, Vol. 24.

[13] Haibin Ling and David Jacobs, (2007) "Shape Classification Using the Inner- Distance", IEEE Transaction on Pattern Analysis and Machine Intelligence, Vol. 29, issue 2.

[14] Thiago R. Trigo and Sergio Roberto M. Pellegrino,(2010) "An Analysis of Features for Hand Gesture Classification”, IWSSIP - 17th International Conference on Systems, Signals and Image Processing.

[15] V. C. Chen, (2004) "Evolution of Bayes, I CA, PCA and SVM Methods for classification", RTO-MPSET-080.

\section{Authors}

Kshama Fating has received B.E. degree in Computer Engineering in 2003.She is currently pursuing M.E. degree at Pune Institute of Computer Technology in Pune University, INDIA.

Archana Ghotkar has received B.E degree in Computer Science and Engineering in 1998 and M.E degree in Computer Engineering in 2000. She is currently pursuing Ph.D in Computer Engineering at university of Pune. She has been an associate professor at Pune Institute of Computer Technology in Pune University, INDIA since 2010. Her main research interest includes computer vision, pattern recognition and human computer interaction. She is a member of ISTE and CSI.
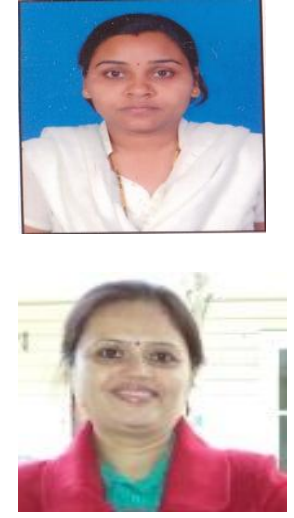